\title{
INTEGRABILITY-PRESERVING REGULARIZATIONS OF LAPLACIAN GROWTH
}

\author{
RAZVAN TEODORESCU*
}

\begin{abstract}
The Laplacian Growth (LG) model is known as a universality class of scale-free aggregation models in two dimensions, characterized by classical integrability and featuring finite-time boundary singularity formation. A discrete counterpart, Diffusion-Limited Aggregation (or DLA), has a similar local growth law, but significantly different global behavior. For both LG and DLA, a proper description for the scaling properties of long-time solutions is not available yet. In this note, we outline a possible approach towards finding the correct theory yielding a regularized LG and its relation to DLA.
\end{abstract}

Mathematics Subject Classification. 30D05, 30E10, 30E25.

Received December 17, 2018. Accepted July 16, 2019.

\section{INTRODUCTION}

There is a large number of models for physical processes involving moving boundaries in two dimensions which can be reduced to the same universality class, known either as Hele-Shaw processes or Laplacian Growth (LG). Examples of such physical phenomena include: electrodeposition [77], solidification [42], viscous fingering [7], bacterial and cancer cells growth [6], to illustrate only a few of the experimental areas where this model is widely used. The mathematical formulation of this dynamical system can be traced back many decades ago, to the pioneering work of Polubarinova-Kochina and Galin [19,67] and has since generated a lot of interest, owing in particular to its applicability to a large number of different research fields (see [23] and references therein), such as: propagation of crystallization fronts, dielectric breakdown and structural failure (shock) propagation, the stochastic Löwner equation, inverse spectral problems for non-normal operators, integrable hierarchies, shape recognition and domain reconstruction in computer tomography, and the list could continue.

Considered from a fluid dynamical point of view, the process can be described as follows. In two dimensions, two incompressible and immiscible fluids (one viscous and the other inviscid) are separated by an infinitely-thin boundary (modeled by a real-analytic Jordan curve). The dynamics of the boundary is governed by Darcy's law, according to which the normal component of the boundary velocity is proportional to the normal component of the pressure gradient. If, due to external factors (or to initial conditions), the interface develops a region of high curvature (a "bump") at some point in time, the pressure gradient on that bump will be larger than on the rest of the boundary. The bump therefore will be moving ("growing") faster than the flatter portions of the boundary, and will be quickly amplified during the growth process. The growth process is thus very unstable and may produce asymptotic shapes such as long, thin "fingers" [14, 81] or, in the long time limit, a fractal-like

Keywords and phrases: Integrable systems, free boundary problem, quadratic differentials.

4202 E. Fowler Ave., CMC342, Tampa, FL 33620, USA.

* Corresponding author: razvan@usf.edu 
cluster with characteristics similar to those obtained in the Diffusion Limited Aggregation (DLA) processes, which is a diffusion-governed variant of the same growth law, cf., e.g., [6, 70, 77].

The mathematical model governing these processes reduces to the following equation for the dynamics of the moving boundary:

$$
V(\zeta)=\partial_{n} G_{D(t)}(\zeta, a)
$$

Here $V$ is the normal component of the velocity of the moving boundary $\partial D(t)$ of the time-dependent domain $D(t) \subset \mathbb{C}, \zeta \in \partial D(t), t$ is time, $\partial_{n}=\widehat{N} \cdot \vec{\nabla}_{\zeta}$ is the normal component of the gradient, and $G_{D(t)}(\xi, a)$ is the Dirichlet Green's function of the domain $D(t)$ for the Laplace operator with a unit source located at the point $a \in D(t)$. Equation (1.1) is known as the Darcy law (in recognition of the original, empirical law derived by Darcy in describing the effective flow of water through porous solids, under the influence of gravity, $c f$. [23] for details).

In DLA, a random walker is released from $a=\infty$, and wanders into $D(t \in \mathbb{N})$ with absorbing boundary conditions, with probability of first-time exit through a subset $\gamma$ of the boundary $\partial D(t)$ given by the harmonic measure $\mathrm{d} \omega(\gamma, a)$. The discrete time $t$ is then incremented by one unit, and the process is repeated.

The contribution of Polubarinova-Kochina and Galin to the theory led to the reformulation of this problem as the area-preserving diffeomorphism identity

$$
\Im\left(\bar{f}_{t} f_{\theta}\right)=1
$$

where $f(t, \cdot)(\mathbb{T})=\partial D(t)$ is the moving boundary (assumed smooth), parameterized by $\theta \in \mathbb{T}=\partial \mathbb{D}$, and conformal when analytically extended into the region $\Im \theta \geq 0$ [19,67]. Equation (1.2) is associated to remarkable properties, such as the existence of a countably-infinite set of conservation laws:

$$
C_{n}(t)=\frac{1}{\pi} \int_{D(t)} z^{n} \mathrm{~d} x \mathrm{~d} y=C_{n}(0)
$$

where $n \in \mathbb{Z}$ is $n \geq 0$ [74] (or $n \leq 0$ [57]) in the case of an finite (infinite) domain $D(t)$, and exact time-dependent closed-form solutions [87].

Unfortunately, as shown in [23] and many other works, this dynamical process is known to develop boundary singularities in finite time, which do not admit a continuation in the space of strong (classical) solutions. While the problem of existence of an infinite-time strong solution (except for the simple geometry corresponding to homothetic ellipsoidal domains [87]) is open, there is a large class of boundaries for which evolution towards a singularity in finite time has been established [23].

This paper is organized as follows: in the next section, a summary review of recent attempts at regularizing this dynamical process with infinite-dimensional symmetry group (both for LG and DLA) is provided. This survey discusses both the mathematical component and the physical interpretation of the works reviewed. In Section 3, a new proposal for a possible solution is introduced. Based on the weak formulation of LG, this approach has the advantage that a solution is available for all times, while the physical interpretation (in the sense of quantum deformations) relates the new formulation to the concept of quantum phase transitions. A brief comments section concludes this paper.

The author wishes to thank the hospitality of the Mathematical Institute at Köln University, where this work was initiated in May 2018.

\section{INTEGRABILITY-PRESERVING REGULARIZATION: A BRIEF HISTORY}

There have been several different attempts at identifying a more general dynamical system which would naturally incorporate LG and DLA as special limits, retaining the integrability structure of LG while remaining compatible with the rescaling invariance of DLA. The difficulty can be easily uncovered, from each of these two 
points of view: as an integrable system, LG is equivalent to the dispersionless limit of the Toda hierarchy [80], and therefore does not have any special lenthgscale, which is another way of saying that its integrable structure is conformal in nature. In order to provide a (short-scale) regularization mechanism, it would appear that this symmetry needs to be broken, which would do away with all the features that make LG an interesting process to begin with.

On the other hand, DLA is in turn marked by its formulation as a discrete aggregation process, and by the difficulty of defining probability measures on spaces of curves. When attempting to describe DLA by starting from purely discrete probability measures (such as in the recent works [1-3]), the main difficulty stems from the fact that do not have yet a generalized Central Limit Theorem for aggregation processes of these types.

The need for finding an extension to both LG and DLA is due to the inherent limitations of both of these models, when trying to use them to describe complex growth patterns occurring in nature. For example, it is known from experiments that $2 \mathrm{D}$ growth processes governed by the boundary Darcy law do not exhibit finite-time singularities, but instead the process continues by means of viscous fingering, which converts a highcurvature local instability into a branching process [70]. However, classical solutions for LG with the same type of initial conditions cannot be continued past a boundary singularity formation [27].

Likewise, while computer simulations of DLA can successfully produce approximations to various fractal dimensions of the DLA cluster, a rigorous theory for this process is not available yet, despite several attempts at introducing a proper short-scale regularization by a number of mechanisms [13, 32, 33, 65].

We briefly survey the recent attempts at describing either (or both) LG and DLA as special limits of an integrable dynamical process with a short length-scale regularization, after which we provide an alternative approach.

\subsection{Normal random matrix ensembles}

In [83] and subsequent publications, LG was linked to the equilibrium distribution of an ensemble of normal random matrices, in a prescribed external field. The Normal Random Matrix Ensemble is defined through the statistical weight associated with a function $V\left(M, M^{\dagger}\right)$, where $\left[M, M^{\dagger}\right]=0$, so that both $M$ and $M^{\dagger}$ can be diagonalized simultaneously:

$$
d \mathbb{P}(M)=e^{-N \operatorname{tr} V\left(M, M^{\dagger}\right)} \mathrm{d} \mu(M) .
$$

Here $N \in \mathbb{N}, N \gg 1$ is a parameter describing the size of matrices considered, and the measure of integration over normal matrices is induced by the flat metric on the space of all complex matrices $d_{C} M$, where $d_{C} M=$ $\prod_{i j} d \operatorname{Re} M_{i j} d \operatorname{Im} M_{i j}$. Using a standard procedure, one passes to the joint probability distribution of eigenvalues $z_{1}, \ldots, z_{N}$ :

$$
d \mathbb{P}\left(\left\{z_{1}, \ldots, z_{N}\right\}\right)=\frac{1}{\tau_{N}}\left|\Delta_{N}(z)\right|^{2} \prod_{j=1}^{N} e^{-N V\left(z_{j}, \bar{z}_{j}\right)} d^{2} z_{j}
$$

Here $d^{2} z_{j} \equiv d x_{j} d y_{j}$ for $z_{j}=x_{j}+i y_{j}, \Delta_{N}(z)=\operatorname{det}\left(z_{j}^{i-1}\right)_{1 \leq i, j \leq N}=\prod_{i>j}^{N}\left(z_{i}-z_{j}\right)$ is the Vandermonde determinant, and

$$
\tau_{N}=\int\left|\Delta_{N}(z)\right|^{2} \prod_{j=1}^{N} e^{-N V\left(z_{j}, \bar{z}_{j}\right)} d^{2} z_{j}
$$

is a normalization factor, the partition function of the matrix model (also called tau ( $\tau$ )-function). It is in fact the tau-function $\tau_{N}(t)$ of the 2D Toda hierarchy, whose large $N$ limit describes the solution of the Laplacian 
growth equations [90],

$$
\tau_{N}(t)=\int \ldots \int \prod_{i \neq j}\left|z_{i}-z_{j}\right|^{2} e^{-N \sum_{k=1}^{N} V\left(z_{k}\right)} d^{2} z_{1} \cdots d^{2} z_{N},
$$

in the case when the function $V(z)$ has the form

$$
V(z)=\frac{1}{t_{0}}\left(|z|^{2}-Q(z)-\overline{Q(z)}\right), \quad Q(z)=\sum_{k=2}^{d+1} t_{k} z^{k}, \quad t_{k} \in \mathbb{R} .
$$

The parameter $t>0$ is a time variable. At the same time, the right hand side of (2.4) represents the partition function of the distribution of eigenvalues in a normal matrix model, hence the key problem is to study the large $N$ limit of the multiple integral in the right hand side of equation (2.4). The eigenvalue density is given by

$$
\rho_{N}(z) \equiv \frac{1}{\tau_{N}} \int \sum_{j=1}^{N} \delta\left(z-z_{j}\right)\left|\Delta_{N}(z)\right|^{2} \prod_{j=1}^{N} e^{-N V\left(z_{j}, \bar{z}_{j}\right)} d^{2} z_{j}
$$

Assume that we are given a domain $D$ of normalized area $t$ and harmonic moments (1.3) $t_{k}=\frac{C_{k}}{k}, k \geq 1$, and the function $V(z)$ satisfies the constraint

$$
\int_{\mathbb{C}}|z|^{n} e^{-N V(z)} d^{2} z<\infty \quad n=0,1,2, \ldots
$$

for all for values of the scaling parameter $N>0$. For fixed $N \in \mathbb{N}$, the orthogonal polynomials $\left\{P_{n}^{(N)}(z)\right\}$ with weight function $e^{-N V(z)}$ are defined by

$$
\int_{\mathbb{C}} P_{n}^{(N)}(z) \overline{P_{m}^{(N)}(z)} e^{-N V(z)} d^{2} z=\delta_{n m}
$$

The approximation method presented in this approach is based on the following statement: as $n \rightarrow \infty, N \rightarrow$ $\infty, n \rightarrow N t, n \leq N$, the squared norms of weighted polynomials $\left|P_{n}^{(N)}(z)\right|^{2} e^{-N V(z)}$ (which we denote by $\mu_{n}^{(N)}(z)$ in the following) converge to the harmonic measure of the domain $D(t)$, with support $\Gamma(t)=\partial D(t)$ :

$$
\mu_{n}^{(N)}(z)=\left|\left(f^{-1}(z)\right)^{\prime}\right|\left[1+O\left(\frac{1}{N}\right)\right], n, N \rightarrow \infty
$$

where $f(z)$ maps conformally the exterior of the unit disc to the exterior of the domain $D(t)$.

While this approach does indeed provide a properly regularized, integrability-preserving deformation of LG, it has not produced so far solutions that could explain the mechanism of viscous fingering, with the notable exception of the special compressibility shock solutions of [44-46], for which a different physical interpretation is available. However, as indicated in Section 3, this approach does lead to a different class of solutions, at the cost of abandoning the original boundary dynamics as the primary formulation of the dynamical process. 


\subsection{Discretized "path integrals" and electrostatic clusters: DLA models}

\subsubsection{DLA-like models as aggregation of electrostatic clusters}

Consider the dynamical process given by the time-dependent family of conformal maps sending $\mathbb{D}$ into $\Omega(t)$, and satisfying

$$
\frac{\partial f(z, t)}{\partial t}=z f^{\prime}(z, t) \frac{1}{2 \pi} \int_{\mathbb{T}} \frac{z+\zeta}{z-\zeta} \frac{|\mathrm{d} \zeta|}{\left|f^{\prime}(\zeta, t)\right|^{\alpha}}
$$

where $\alpha \in \mathbb{R}$ is a model parameter. As shown in [7,13], (2.9) can be related to the Dirichlet Green's functions $G(z, t)$ of the domains $\Omega(t)$, with a source at $\infty$ : if $V_{n}$ denotes the normal velocity of the boundary, then $V_{n} \sim|\nabla G(\cdot, t)|^{\alpha-1}$, therefore describing a more general class of LG-like processes known as Dielectric Breakdown Models (DBM).

For $\alpha=2$ in (2.9), we retrieve a formulation of Laplacian Growth, as shown, e.g., in [23] and references therein. The limiting case $\alpha=0$ reduces (after applying the Poisson integral formula with constant Dirichlet data) (2.9) to

$$
\partial_{t} f(z, t)=z f^{\prime}(z, t)
$$

for which a class of solutions is given by $f(z, t)=\varphi\left(e^{t} z\right)$, for any conformal map $\varphi$. As the local growth law has velocity inversely proportional to the curvature, any initial real-analytic boundary will be smoothed out further by the process, with the curvature converging to a constant. In particular, the solution $f(z, t)$ exists for all $t>0$, and the infinite-time limiting shape is a circle.

As discussed in [33] (from where this argument is borrowed), there is another case where (2.9) can be solved for all $t>0$ : setting an arbitrary $\alpha \in \mathbb{R}$, and the initial condition $\varphi(z)=z$, then

$$
f(z, t)=(1+\alpha t)^{\frac{1}{\alpha}} z
$$

solves the initial-value problem, and the solution is again smooth, for all $t>0$ when $\alpha \geq 0$, and for $t<-1 / \alpha$ for negative $\alpha$.

The conformal maps in (2.10) correspond to the small-particle scaling limit for the Hastings-Levitov model with strong regularization [25]. The authors conjectured that the growth behavior in the Hastings-Levitov random aggregation model will exhibit a phase transition at the parameter $\alpha=1$, indicating the separation between boundary dynamics dominated by smoothing (for $\alpha<1$ ) and curvature-driven instability (for $\alpha>1$ ).

Starting from this formulation for the DBM class of dynamics, various ad-hoc regularizations were introduced, e.g. by adding a small parameter $\sigma>0$ to $f^{\prime}(t, z)$ under the integral sign in (2.9), which basically shifts the boundary curves away from the level lines of the Green's function (for the simplest case $\alpha=0$ ), therefore preventing the velocity (left-hand side of (2.9)) from diverging. However, the selection and even asymptotic limit $(\sigma \rightarrow 0)$ of the new parameter seem to be important for the type of regularized solutions obtained, which is indicative of the singular-perturbation character of LG.

The main open problem that is not addressed by this formulation is the branching behavior observed in numerous computer simulations of DLA. The theory does not seem to have any mechanism for this type of instability (which corresponds to the viscous fingering of LG), and therefore cannot provide a method for computing the fractal dimensions of large DLA clusters.

\subsubsection{Probabilistic models for DLA and asymptotic theory}

In [1-3], an alternative approach to defining DLA was provided, based on the expected weak convergence of probability functions describing a discretized boundary, and starting from a staking process for identical objects 
of given size, which is then rescaled in a prescribed manner. We reproduce here the main idea of this approach, referring the reader to the works cited for more details.

To define the growth process, the unit circle is partitioned into $N \gg 1$ equal segments of length $\Delta \phi=\frac{2 \pi}{N}$. Their images on the boundary of the cluster, under the conformal map $f(z, t)$, will have lengths

$$
l_{k}=\left|f^{\prime}\left(z_{k}, t\right)\right| \Delta \phi=\frac{2 \pi}{N}\left|f^{\prime}\left(z_{k}, t\right)\right|,
$$

where $z_{k}=e^{i \phi_{k}}$, where $\phi_{k} \in[k \Delta \phi,(k+1) \Delta \phi]$. The process consists of rectangular "blocks", each landing on the segments $\gamma_{i}=f(., t)([i \Delta \phi,(i+1) \Delta \phi]) \subset \Gamma(t), i=1,2, \ldots, N$. A block landing on the $\mathrm{i}$-th segment is prescribed the "height"

$$
h_{i}=\frac{\epsilon}{l_{i}}
$$

where $0<\epsilon \ll 1$ is a regularization parameter, thus ensuring equal areas.

Assume now that during a single time step $k_{i} \geqslant 0$ blocks land on the segment $i$. Then the growth step is fully specified by the set of $N$ non-negative integers $k_{i}$ (occupation numbers). For a given total number of blocks, $K$, the occupation numbers satisfy the constraint

$$
\sum_{i=1}^{N} k_{i}=K .
$$

If all the building blocks attach to the cluster independently, the probability of a particular growth step $\left\{k_{i}\right\}_{i=1}^{N}$ is given by the multinomial distribution,

$$
P\left(\left\{k_{i}\right\}\right)=\frac{K !}{N^{K} \prod_{i=1}^{N} k_{i} !} .
$$

According to the authors, the displacement $\delta n_{i}$ of the segment $\gamma_{i}$ in one time step is computed as:

$$
\delta n_{i}=k_{i} h_{i}=\epsilon \frac{k_{i}}{l_{i}}=\frac{\epsilon N}{2 \pi} \frac{k_{i}}{\left|f_{t}^{\prime}\left(z_{i}\right)\right|}
$$

Identifying the displacement (per unit of discrete time) to velocity, the corresponding Darcy-type growth law becomes

$$
k_{i}=\frac{2 \pi}{\epsilon N}\left|f_{t}^{\prime}\left(z_{i}\right)\right| v_{i}
$$

where $v_{i}=\delta n_{i}$ is the normal "velocity" of the boundary.

The family of models is parameterized by $\kappa:=\frac{K}{N}$, understood as the average number of building blocks deposited to a single segment on the boundary. It is claimed that the limit $\kappa \ll 1$ corresponds to DLA.

Independently, a second model parameter is the "area" of a building block, $\epsilon$, with the limit case $\epsilon \rightarrow 0$ yielding LG.

The discrete-time process described above is related to a continuous-time stochastic process $X_{t}(\phi)$ by taking the limit (in distribution) of the random variable $\frac{k_{i}}{\kappa}$, as $N \rightarrow \infty, K \rightarrow \infty$, at a fixed value of $\kappa, \epsilon$.

After computing a Freidlin-Wentzell type of functional for large deviations of the process $X_{t}$ from its mean $\mathbb{E}\left(X_{t}\right)=1$, various limits are considered, such as $\epsilon \rightarrow 0$ (supposed to lead to a deterministic process equivalent to $\mathrm{LG}$ ) and $\kappa \rightarrow \infty$ (for which the large deviations functional becomes the Cramér functional for a Poisson 
process). It is expected that an application of Varadhan's principle for this functional will, for the proper choice of parameters $\epsilon, \kappa$, yield DLA as the solution to a variational problem.

\subsection{Quantum deformations and representation theory for CFT}

In [8], a symmetry-based approached was discussed as a potential way of embedding LG (and, supposedly, DLA) into a wider integrable system, whose representations would incorporate finite-dimensional reductions corresponding to those of rational conformal field theories (RCFT), as described in many works (see, e.g., [23], Sects. 7-8). The justification for this approach lies in the identification of LG with the dispersionless Toda hierarchy, the Gelfand-Dickey hierarchy, and the KP hierarchy $[23,80,83,90]$. As the latter is known to contain the Virasoro algebra (at arbitrary values of the central charge), the connection with RCFT is to be expected.

However, such an embedding has not been established rigorously, yet. The main obstacle for this approach stems from the limitations of quantum deformations, as discussed briefly in the following. On one hand, the most general procedure for constructing quantum deformations is the Drinfeld method ([21], Sect. 6), which yields a quantum double (pair of Hopf algebras) for every compact Lie group (classical symmetry). In this approach, an (arbitrary) deformation parameter is naturally introduced, which should appear as the desired regularization parameter needed in order to resolve the singularities emerging in the classical (undeformed) problem. The limitation is due to the fact that the method is restricted to classical symmetries which correspond to finite-dimensional Lie groups, unlike the case of LG.

In those situations where LG dynamics can be mapped, locally, to a finite-dimensional reduction of an integrable hierarchy, the procedure works as expected. For example, boundary singularities corresponding to reductions to an elliptic curve (known as the (3,2)-cusps) were considered in [46] and [84], leading to the KdV and Painlevé I equations, respectively. This is an illustration of the well-known fact that integrable deformations of the field of elliptic functions lead to the Painlevé I transcendent [46], which is also a scaling reduction of the $\mathrm{KdV}$ equation [84].

Another classical-quantum relationship was considered in [8], based on the observation that (again, in the case of quantum deformations corresponding to an elliptic curve) certain classical Poisson structures are naturally embedded into a Sklyanin algebra, depending on an auxiliary parameter, i.e. the modulus of the elliptic curve.

The difficulty in applying such a program to LG in general is due to the incomplete classification of boundary singularities arising from the classical dynamics. In the absence of a probabilistic description of various types of possible boundary singularities (e.g., it is known that classical solutions cannot be continued in the cases of cusps of types $(4 k-1,2), k \in \mathbb{N}$, [23], but the likelihood of any such cusp formation has not been quantified), it is not possible to apply the deformation method in order to compute fractal dimensions and critical exponents.

In the remaining of the paper, we investigate another approach towards an integrable deformation of LG, and its application to the case of boundary singularity formation.

\section{WEAK SOLUTIONS AND QUANTIZATION}

\subsection{Equilibrium measures and orthogonal polynomials}

\subsubsection{The classical equilibrium problem for logarithmic potentials on $\mathbb{C}$}

Let $\sigma$ be a finite Borel measure on the plane, and define its associated logarithmic potential $\Phi^{\sigma}(z) \equiv$ $-\int \log |z-x| \mathrm{d} \sigma(x)$, logarithmic energy $W[\sigma] \equiv-\iint \log |z-x| \mathrm{d} \sigma(x) \mathrm{d} \sigma(z)$, both not necessarily finite. Denoting by $\operatorname{supp}(\sigma)$ the support of $\sigma$, consider an admissible real-valued function $V$, called external field. Accordingly, we obtain the total potential

$$
U^{\sigma, V}(z) \equiv \Phi^{\sigma}(z)+V(z),
$$


and the total energy

$$
\mathcal{E}[\sigma, V]=W[\sigma]+2 \int V(z) \mathrm{d} \sigma(z)
$$

Given a class of measures (e.g., atomic, absolutely continuous, defined on a compact, etc.), we define the equilibrium measure as the minimizer, within that class, of the total energy $\mathcal{E}[\sigma, V]$ (including for the case $V=0$, in which case the equilibrium measure is a characteristic of the set on which it is supported).

For example, given a compact set $K \subset \mathbb{C}$, its equilibrium measure (when it exists) is known as the Robin measure of $K$. Denoted $\omega_{K}$, this measure is unique, supported on the outer boundary of $K$, and its energy is given by

$$
W\left[\omega_{K}\right]=-\ln \operatorname{cap} K
$$

where cap K is the logarithmic capacity of $K$ (discussed in some detail in the next subsection). However, the equilibrium measure in a (non-trivial) external field is a much more complicated object. In particular, it is not know a priori where its support will be located.

\subsubsection{Equilibrium measures for random matrix ensembles}

Let $t>0$ and choose a closed subset $\Sigma \subset \mathbb{R}$. If we denote by $\mathbb{M}_{t}(\Sigma)$ the set of positive Borel measures $\sigma$ compactly supported in $\Sigma$ with total mass $t$, for a real-analytic external field $V(x)$ on $\mathbb{R}$, the subset of measures $\sigma \in \mathbb{M}_{t}$ with finite energy is not empty (see [76], Chap. I). Therefore, there exists a unique minimizer measure, denoted $\mu_{t}(V) \in \mathbb{M}_{t}$, such that

$$
\mathcal{E}\left[\mu_{t}, V\right]=\min _{\sigma \in \mathbb{M}_{t}} \mathcal{E}[\sigma, V]
$$

If the function $V(x)$ (sometimes called "confining potential" due to its physical interpretations) grows at infinity faster than $\log |x|$, then $\operatorname{supp}\left(\mu_{\mathrm{t}}\right)$ is a compact set in $\mathbb{R}$ for all $t>0$, and $\mu_{t}$ is completely determined by the equilibrium condition satisfied by the total potential

$$
U^{\mu_{t}, V}(z)=u_{t}, \forall z \in \operatorname{supp}\left(\mu_{t}\right), U^{\mu_{t}, V}(z) \geq u_{t} \forall z \in \mathbb{R},
$$

where the constant $u_{t}$ is given by

$$
u_{t}:=\frac{1}{t}\left(\mathcal{E}\left[\mu_{t}, V\right]-\int V(x) \mathrm{d} \mu_{t}(x)\right) .
$$

An application of the notion of equilibrium measures for orthogonal polynomials and random matrix models [9-12] is based on the following fundamental result from [22, 56] (see also [47, 76]): for a fixed external field $V$ and $N>n>0$, let $(.,$.$) be the scalar product on C(\mathbb{R})$, with measure $e^{-2 N V(x)} d x$, and induced norm $\|$. $\|$, and define the orthogonal monic polynomials $Q_{n}^{(N)}$, such that $\left(Q_{n}^{(N)}, x^{k}\right)=0, \quad k=0,1, \ldots, n-1$. These polynomials are associated with $n \times n$ Jacobi matrices, as a representation of their recurrence relations. For $n, N \rightarrow \infty$ at fixed $\frac{n}{N}=t>0$, the zero-counting measure $\chi_{n}:=\frac{1}{n} \mathbb{I}_{\left\{Q_{n}^{(N)}(x)=0\right\}}$ converges (in the weak star topology) to the equilibrium measure $\mu_{t}(V) \in \mathbb{M}_{t}$, which is also the limit spectrum of the infinite Jacobi matrix, associated with the scaling $\frac{n}{N} \rightarrow t$. Moreover, $\frac{\log \left\|Q_{n}^{(N)}\right\|}{N}$ has the non-zero limit $u_{t}$, the equilibrium potential constant.

The connection to random matrix ensembles arises when considering the class of uniform atomic distributions. Denote by $M_{n}$ the class of such distributions supported on $n$ points on the real line $\zeta=\left(\zeta_{1}, \ldots, \zeta_{n}\right) \in \mathbb{R}^{n}$, which 
can be identified to the atomic distribution itself, $\zeta \in \mathbb{R}^{n}=\nu_{n} \in M_{n}$, then the corresponding discrete energies are defined by $(c f .(3.2))$

$$
E[\boldsymbol{\zeta}, V]:=E\left[\nu_{n}, V\right]:=\sum_{i \neq j} \log \frac{1}{\left|\zeta_{i}-\zeta_{j}\right|}+2 \int V(\zeta) \mathrm{d} \nu_{n}(\zeta)
$$

Introducing the joint atomic probability mass density

$$
d \mathbb{P}_{n}^{(N)}(\boldsymbol{\zeta})=\frac{1}{Z_{n}^{(N)}} e^{-E[\boldsymbol{\zeta}, N V]} \mathrm{d} \boldsymbol{\zeta}, \quad Z_{n}^{(N)}=\int_{\mathbb{R}^{n}} e^{-E[\boldsymbol{\zeta}, N V]} \mathrm{d} \boldsymbol{\zeta}
$$

we first notice that it is identical to the probability density for eigenvalue distribution of the normal random matrix model, when the spectrum is restricted to the real line (which, in fact, describes the so-called the Gaussian Unitary Ensemble, perturbed by the potential function $N \cdot V(x)$ ). Then the classical Heine formula $Q_{n}^{(N)}(x)=\int_{\mathbb{R}^{n}} \prod_{k=1}^{n}\left(x-\zeta_{k}\right) d \mathbb{P}_{n}^{(N)}(\boldsymbol{\zeta})$ shows that the orthogonal polynomial $Q_{n}^{(N)}$ is the average of polynomials with real zeros randomly distributed according to the probability density $d \mathbb{P}_{n}^{(N)}$.

\subsection{Minimal capacity sets in external fields and moduli functions}

We review the main results characterizing minimal capacity sets in $\mathbb{C}$, and how they relate to the problem of finding the support of equilibrium measures, following [23]. Let $\Omega$ be a domain in $\mathbb{C}$ and $\rho(z)$ be a real-valued, Borel measurable, non-negative function in $L^{2}(\Omega)$, defining a differential metric $\rho$ on $\Omega$ by $\rho:=\rho(z)|\mathrm{d} z|$. For any locally rectifiable curve $\gamma \subset \Omega$, the integral $l_{\rho}(\gamma):=\int_{\gamma} \rho(z)|\mathrm{d} z|$, is called the $\rho$ - length of $\gamma$, while the $\rho-$ area of $\Omega$ is given by

$$
A_{\rho}(\Omega):=\iint_{\Omega} \rho^{2}(z) \mathrm{d} x \mathrm{~d} y .
$$

If $\Gamma$ is a family of curves $\gamma$ in $\Omega$, we define the modulus of $\Gamma$ in $\Omega$ by

$$
m(\Omega, \Gamma)=\inf _{\rho} \frac{A_{\rho}(\Omega)}{L_{\rho}^{2}(\Gamma)}, L_{\rho}(\Gamma):=\inf _{\gamma \in \Gamma} l_{\rho}(\gamma)
$$

where $L_{\rho}(\Gamma)$ is the $\rho$-length of the family $\Gamma$, and the infimum is taken over all metrics $\rho$ in $\Omega$.

The modulus is conformally-invariant, and monotonic relative to inclusion of curve families: let $\Gamma$ be a family of curves in a domain $\Omega \in \overleftrightarrow{\mathbb{C}}$, and let $w=f(z)$ be a conformal map of $\Omega$ onto $\widetilde{\Omega} \in \widehat{\mathbb{C}}$. If $\widetilde{\Gamma}:=f(\Gamma)$, then $m(\Omega, \Gamma)=m(\widetilde{\Omega}, \widetilde{\Gamma})$. Also, if $\Gamma_{1} \subset \Gamma_{2} \subset \Omega$, then $m\left(\Omega, \Gamma_{1}\right) \leq m\left(\Omega, \Gamma_{2}\right)$.

\subsubsection{Capacity and reduced modulus}

Let $\Omega \subset \widehat{\mathbb{C}}$ be a simply connected domain, and $a \in \Omega, a \neq \infty$. For the doubly connected domain $\Omega_{\epsilon}=$ $\Omega \backslash B_{a}(\epsilon), 0<\epsilon \ll 1$, the reduced modulus of $\Omega$ with respect to the point $a$ is defined as

$$
M(\Omega, a):=\lim _{\epsilon \rightarrow 0}\left(M\left(\Omega_{\epsilon}\right)+\frac{1}{2 \pi} \log \epsilon\right)
$$

where $M\left(\Omega_{\epsilon}\right)$ is the modulus of the domain $\Omega_{\epsilon}$ with respect to the family of curves that separate its boundary components. As shown in [31], it is also equal to $\frac{1}{2 \pi} \log R(\Omega, a)$, where $R(\Omega, a)$ is the conformal radius of $\Omega$ with respect to $a$. 
The reduced modulus $M(\Omega, \infty)$ of a simply connected domain $\Omega$, with respect to $\infty \in \Omega$ is defined as the reduced modulus of the image of $\Omega$ under the map $1 / z$,

$$
M(\Omega, \infty)=-\frac{1}{2 \pi} \log R(\Omega, \infty)
$$

For two disjoint compact sets $K_{1}, K_{2}$ in $\mathbb{C}$, the capacity of the complement (often called condenser, owing to the connections to electrostatics) is defined as $\operatorname{cap}\left(\widehat{\mathbb{C}} \backslash\left(K_{1} \cup K_{2}\right)\right):=\inf \mathcal{E}_{D}\left(\widehat{\mathbb{C}} \backslash\left(K_{1} \cup K_{2}\right)\right)$, where $\mathcal{E}_{D}$ stands for Dirichlet energy, and the infimum is taken over all Lipshitz-continous functions on the set.

A condenser is said to be admissible if there exists a solution to the Dirichlet problem on $\widehat{\mathbb{C}} \backslash\left(K_{1} \cup K_{2}\right)$, continuous on $\widehat{\mathbb{C}}$, taking the value 0 on $K_{1}$ and 1 on $K_{2}$. Therefore, for admissible condensers, the capacity is a conformal invariant. This can be used to compute it for the case when $K_{1}$ and $K_{2}$ are two disjoint continua (compact and connected), yielding cap $C=2 \pi / \log R$, where $R>1$ corresponds to the conformal mapping of $\widehat{\mathbb{C}} \backslash\left\{K_{1} \cup K_{2}\right\}$ onto an annulus $1<|w|<R$.

Let now $K$ be a compact set in $\mathbb{C}$, and condensers of special type $C_{R}=\{|z| \geq R, K\}$ for $R \rightarrow \infty$. The function $\frac{1}{\operatorname{cap} C_{R}}-\frac{1}{2 \pi} \log R$ increases with increasing $R$ and the limit

$$
\operatorname{cap} K=\lim _{R \rightarrow \infty} R \exp \left(-\frac{2 \pi}{\operatorname{cap} C_{R}}\right)
$$

exists and is said to be the logarithmic capacity of the compact set $K \subset \mathbb{C}$. The result (3.9) is known as Pfluger's theorem ([68], Sect. 9).

Next we briefly summarize the definition and some properties of the logarithmic capacity of a compact set $K \subset \mathbb{C}$ following Fekete. For $n=2,3, \ldots$ we consider

$$
\Delta_{n}(K)=\max _{z_{1}, \ldots, z_{n} \in K} \prod_{1 \leq k<j \leq n}^{n}\left|z_{k}-z_{j}\right| .
$$

The maximum exists and is attained for so-called Fekete points $z_{k}^{(n)} \in \partial K, k=1, \ldots, n$. Its value is equal to the Vandermonde determinant

$$
\Delta_{n}(K)=\left|\operatorname{det}_{j, k=1, \ldots, n}\left(z_{k}^{(n)}\right)^{j-1}\right|
$$

Then, the limit

$$
\lim _{n \rightarrow \infty}\left(\Delta_{n}(K)\right)^{\frac{2}{n(n-1)}}=\operatorname{cap} K
$$

exists (see [68]), is known as the transfinite diameter of $K$, and is equal to its logarithmic capacity.

This result establishes the connection between the logarithmic capacity of a compact set (support of the equilibrium measure) (3.3) and the $n \rightarrow \infty$ limit of random matrix ensembles, as discussed in the previous section.

\subsection{Weak solutions of LG and equilibrium measures}

Given a solution to LG for some initial data, in the form of a family of domains $\{\Omega(t)\}_{t \in\left[t_{0}, T\right]}$ with the strict inclusion property $\Omega(s) \subset \Omega(t), \forall t_{0} \leq s<t \leq T$, consider its associated Riemann surface ([63], Sect. 5), and its set of branch points, denoted by $\boldsymbol{B}(t)$. For this data, we then solve the (Chebotarev) problem of finding a set of analytic arcs with endpoints in $\boldsymbol{B}(t), K(t)$, and the equilibrium measure supported it, solving (3.4). 
Denote the equilibrium measure by $\mu(t)$ (functionally dependent on $V$ ), then we refer to the family of sets and measures $\left\{(K(t), \mu(t)\}_{t \in\left[t_{0}, T\right]}\right.$ as the weak solution to LG with prescribed branch point data. It should be noted that, owing to the relation between this problem and the inverse balayage of the domains $\{\Omega(t)\}$, we have $K(s) \subseteq K(t), \operatorname{supp} \mu(s) \subseteq \operatorname{supp} \mu(t), \forall t_{0} \leq s<t \leq T, c f$. [63].

Even in the (typical) case when the (strong) LG solution develops cusp boundary singularities for some value of the area, $t_{c}$, therefore forbidding continuation of the strong solution for $t \geq t_{c}$, the weak solution remains well-defined, but the support of the equilibrium measure branches out at the transition through $t=t_{c}[11,46]$.

To describe both the weak solution in potential-theoretical sense and the integrable character of LG, it is useful to define the function

$$
\Psi(z, t ; V):=\exp \left(V(z)+\int \log |z-\zeta| \mathrm{d} \mu_{t}(\zeta)\right), z \notin \operatorname{supp} \mu(t)
$$

It is subharmonic in a neighborhood of $\operatorname{supp}(\mu)$ (excluding the support), and in a neighborhood of $\infty$, and superharmonic in a tubular neighborhood of $\partial \Omega(t)$. Since, up to a prefactor that is independent on $t$, the function is proportional to the ratio

$$
\exp \left(\mathcal{E}\left[\mu_{t}, V(\zeta)+\log |z-\zeta|\right] / \exp \left(\mathcal{E}\left[\mu_{t}, V(\zeta)\right]\right.\right.
$$

that is the limit (in capacity) of the perturbation determinant for the matrix $L(t)$ associated with the spectrum $\mu(t)$. More precisely, in approximation sense, we can first solve the discrete equilibrium measure problem (3.7), which leads to a sequence of matrices $\left\{L_{n}\right\}$, converging weakly (3.10) to the operator $L(t)$. Correspondingly, the ratios of their perturbation determinants will have a limit related to $\Psi(z, t ; V)$.

For values of $t$ corresponding to a classical LG solution, the gradient of $\Psi(z, t ; V)$ will vanish precisely along the boundary $\partial \Omega(t)$, since it is given by the equation $V^{\prime}(z)+C_{\mu_{t}}(z)=0$, where $C_{\mu_{t}}$ is the Cauchy transform of $\mu_{t}$, which coincides with $C_{\Omega(t)}$ in a neighborhood of $z=\infty$. Therefore, it is possible to define a "weak LG boundary" by the set $\left\{\partial_{z} \Psi(z, t ; V)=0\right\}$ for values of $t$ beyond $t_{c}$, which may consist of a union of domain boundaries and analytic arcs (regarded as the singular boundary sets for domains in the limit of empty interior).

Along the support of $\mu_{t}, \Psi(z, t ; V)$ may be assigned the value 0 , as the tangential derivative vanishes. The two limit normal derivatives are equal and opposite signs.

The relation to DLA is obtained by first formulating it as a limit process that amounts to a "randomized" logarithmic capacity problem (and, therefore, reduced modulus $c f$. Sect. 3.2.1). Consider a measure $\mu_{t}$ supported on a continuum $K_{t}$ consisting of a finite set of analytic arcs, in a neighborhood of the origin, and the circle $K_{R}:=\{|z|=R\}$, such that $K_{t} \subset B_{0}(R)$ (the degenerate case corresponds to $K_{t}=\{0\}$ ). Let $D_{t}$ denote the balayage of $\mu_{t}$, and $\varphi_{t}: D_{t}^{c} \rightarrow \overline{\mathbb{D}}^{c}$ the conformal map sending $\infty$ into $\infty$, with real conformal radius. Denote by $\Sigma_{t}(R):=\varphi_{t}\left(K_{R}\right)$, and $P_{t}$ the perimeter of $\Sigma_{t}(R)$. The probability measure $\nu_{t}$ defined on $\partial \mathbb{D}$ by $\nu_{t}(\gamma):=\ell(\widehat{\gamma}) / P_{t}$, where $\ell(\widehat{\gamma})$ is the arclength measure of the $\operatorname{arc} \widehat{\gamma}$, the intersection between $\Sigma_{t}(R)$ and the radial family intersecting $\gamma$, describes a "deterministic" limit of DLA, in the sense that it obeys the same growth law, averaged over an uniform prior distribution over $K_{R}$. More precisely, the growth process is defined by the inverse balayage of the measure $\chi_{D_{t}}+\tilde{\nu}_{t} d t$, where $\tilde{\nu}_{t}$ is the pre-image (under $\varphi_{t}$ ) of the measure $\nu_{t}$, that is $\mu_{t+d t}=\mu_{t}+\lambda_{t}$, with $\lambda_{t}$ the inverse balayage of $\tilde{\nu}_{t} d t$.

The model can be generalized by replacing the uniform measure over $\Sigma_{t}(R)$ by any other probability measure with support on $\Sigma_{t}(R)$. DLA proper is obtained in the limit $R \rightarrow \infty$, sampling with uniform probability over the circle, and replacing the deterministic flow given by the radial family of curves (equivalently, by the vector field given by the Cauchy transform of $\mu_{t}$, before applying the conformal map $\varphi_{t}$ ), by the Itô process given by adding a Wiener process to the field $C_{\mu_{t}}(z)$. 


\section{CONCLUding REMARKS}

The singular perturbation nature of classical LG is the main source of complications when seeking to embed this boundary dynamics process into more general integrable structures. The weak formulation briefly described here has the advantage of connecting at once with a larger class of optimization problems for which post-critical solutions are known to exist (equilibrium measures in the sense of Chebotarev-Gonchar [22]), as well as to free stochastic processes [89] with the infinite divisibility property, which is a promising path towards formulating a quantum (maximum entropy) generalization of the classical problem.

Acknowledgements. The author is grateful to the referees for comments and suggestions which have improved the present work.

\section{REFERENCES}

[1] O. Alekseev and M. Mineev-Weinstein, Stochastic Laplacian growth. Phys. Rev. E 94 (2016) 060103.

[2] O. Alekseev and M. Mineev-Weinstein, Theory of stochastic Laplacian growth. J. Stat. Phys. 168 (2017) 68-91.

[3] O. Alekseev and M. Mineev-Weinstein, Statistical mechanics of stochastic growth phenomena. Phys. Rev. E 96 (2017), 010103.

[4] F. Balogh, M. Bertola, S.Y. Lee and K.D.T.-R. McLaughlin, Strong asymptotics of the orthogonal polynomial with respect to a measure supported on the plane. Preprint arXiv.math-ph.:1209.6366 (2012).

[5] M. Bauer and D. Bernard, 2D growth processes: SLE and Loewner chains. Phys. Rep. 432 (2006) 115.

[6] E. Ben-Jacob, From snowflake formation to the growth of bacterial colonies. Part 2: Cooperative formation of complex colonial patterns. Contempt. Phys. 38 (1997) 205-241.

[7] D. Bensimon, L. Kadanoff, S. Liang, B. Shraiman and C. Tang, Viscous flows in two dimensions. Rev. Mod. Phys. 58 (1986) 977.

[8] E. Bettelheim, Classical and Quantum Integrability in Laplacian Growth. Preprint arXiv:1506.01463 [nlin.PS] (2015).

[9] P. Bleher and A. Its, Semiclassical asymptotics of orthogonal polynomials, Riemann-Hilbert problem, and universality in the matrix model. Ann. Math. 150 (1999) 185-266.

[10] P. Bleher and A.B.J. Kuijlaars, Large n limit of Gaussian random matrices with external source, part I. Commun. Math. Phys. 252 (2004) 43-76.

[11] P. Bleher and A. Kuijlaars, Orthogonal polynomials in the normal matrix model with a cubic potential. Adv. Math. 230 (2012) 1272-1321.

[12] P. Bleher, A. Kuijllars and S. Delvaux, Random matrix model with external source and a constrained vector equilibrium problem. Commun. Pure Appl. Math. 64 (2011) 116160.

[13] L. Carleson and N. Makarov, Aggregation in the plane and Loewner's equation. Commun. Math. Phys. 48 (2001) 538-607.

[14] E. DiBenedetto and A. Friedman, Bubble growth in porous media. Indiana Univ. Math. J. 35 (1986) 573-606.

[15] M. Duits and A.B.J. Kuijlaars, Painlevé I asymptotics for orthogonal polynomials with respect to a varying weight. Nonlinearity 19 (2006) 2211-2245.

[16] P. Ebenfelt, B. Gustafsson, D. Khavinson and M. Putinar eds., Quadrature Domains and Their Applications, The Harold S. Shapiro Anniversary Volume, Birkhäuser, Basel (2005).

[17] P. Elbau and G. Felder, Density of eigenvalues of random normal matrices. Commun. Math. Phys. 259 (2005) $433-450$.

[18] A.S. Fokas, A.R. Its and A.V. Kitaev, The isomonodromy approach to matrix problems in 2D quantum gravity. Commun. Math. Phys. 147 (1992) 395-430.

[19] L.A. Galin, Unsteady filtration with a free surface. Dokl. Akad. Nauk SSSR 47 (1945) 250-253 (In Russian); English trasl., (Dokl.) Acad. Sci. URSS 47 (1945) 246-249.

[20] S. Garoufalidis, A. Its, A. Kapaev and M. Mari-o, Asymptotics of the Instantons of Painlev I. Int. Math. Res. Notices 2012 (2012) 561-606.

[21] C. Gomez, M. Ruiz-Altaba and G. Sierra, Quantum groups in two-dimensional physics. Cambridge Univ. Press (1996).

[22] A.A. Gonchar and E.A. Rakhmanov, Equilibrium measure and the distribution of zeros of extremal polynomials. Mat. Sbornik. 125 (1984) 117-127. Translation from Mat. Sb., Nov. Ser. 134 (1987) 306-352.

[23] B. Gustafsson, R. Teodorescu and A. Vasil'ev, Classical and stochastic Laplacian growth. Springer International Publishing (2014).

[24] A. Hassel and S. Zelditch, Determinants of Laplacians in exterior domains. IMRN (1999) 971-1004.

[25] M.B. Hastings and L.S. Levitov, Laplacian growth as one-dimensional turbulence. Physica D 116 (1998) 244.

[26] H. Hedenmalm and S. Shimorin, Hele-Shaw flow on hyperbolic surfaces. J. Math. Pures Appl. 81 (2002) 187-222.

[27] S.D. Howison, Fingering in Hele-Shaw cells. J. Fluid Mech. 167 (1986) 439-453.

[28] S.D. Howison, Complex variable methods in Hele-Shaw moving boundary problems. Eur. J. Appl. Math. 3 (1992) 209-224.

[29] S. Howison, I. Loutsenko and J. Ockendon, A class of exactly solvable free-boundary inhomogeneous porous medium flows. Appl. Math. Lett. 20 (2007) 93-97. 
[30] A.R. Its and L.A. Takhtajan, Normal matrix models, $\bar{\partial}$-problem, and orthogonal polynomials on the complex plane. Preprint arXiv.math.:0708.3867 (2007).

[31] J. Jenkins, Univalent functions and conformal mapping. Springer-Verlag (1958).

[32] F. Johansson Viklund, A. Sola and A. Turner, Scaling limits of anisotropic Hastings-Levitov clusters. Ann. Inst. Henri Poincaré Probab. Stat. 48 (2012) 235-357.

[33] F. Johansson Viklund, A. Sola and A. Turner, Small-particle limits in a regularized Laplacian growth model. Commun. Math. Phys. 334 (2015) 331-366.

[34] Q. Kang, D. Zhang and S. Chen, Immiscible displacement in a channel: simulations of fingering in two dimensions. Adv. Wat. Res. 27 (2004) 13-22.

[35] L. Karp, Construction of quadrature domains in $R^{n}$ from quadrature domains in $R^{2}$. Complex Var. Theory Appl. 17 (1992) $179-188$.

[36] D. Khavinson, M. Mineev-Weinstein and M. Putinar, Planar eliptic growth. Complex Anal. Oper. Theory 3 (2009) 425-451.

[37] D. Khavinson, M. Mineev-Weinstein, M. Putinar and R. Teodorescu, Lemniscates are destroyed by eliptic growth. Math. Res. Lett. 17 (2010) 337.

[38] I. Kostov, I. Krichever, M. Mineev-Weinstein, P. Wiegmann and A. Zabrodin, $\tau$-function for analytic curves. Vol. 40 of Random Matrix Models and Their Applications, Math. Sci. Res. Inst. Publ. Cambridge University Press (2001) 285-299.

[39] I. Krichever, A. Marshakov and A. Zabrodin. Integrable structure of the Dirichlet boundary problem in multiply-connected domains. Commun. Math. Phys. 259 (2005) 1-44.

[40] I. Krichever, M. Mineev-Weinstein, P. Wiegmann and A. Zabrodin, Laplacian growth and Whitham equations of soliton theory. Physica D 198 (2004) 1-28.

[41] P.P. Kufarev, A solution of the boundary problem of an oil well in a circle. Dokl. Acad. Nauk SSSR. 60 (1948) $1333-1334$.

[42] J.S. Langer, Instabilities and pattern formation in crystal growth. Rev. Mod. Phys. 52 (1980) 1-28.

[43] S.-Y. Lee and N. Makarov, Topology of quadrature domains. arXiv:1307.0487 [math.CV] (2015).

[44] S.-Y. Lee, R. Teodorescu and P. Wiegmann, Shocks and finite-time singularities in Hele-Shaw flow. Physica D 238 (2009) $1113-1128$.

[45] S.-Y. Lee, R. Teodorescu and P. Wiegmann, Weak solution of the Hele-Shaw problem: shocks and viscous fingering. JETP Lett. 92 (2010) 9196.

[46] S.-Y. Lee, R. Teodorescu and P. Wiegmann, Viscous shocks in Hele-Shaw flow and Stokes phenomena of the Painlevé I transcendent. Physica D 240 (2011) 1080-1091.

[47] D.S. Lubinsky, H.N. Mhaskar and E.B. Saff, A proof of Freud's conjecture for exponential weights. Constr. Approx. 4 (1988) $65-83$.

[48] E. Lundberg and D. Khavinson, Gravitational lensing by a collection of objects with radial densities. Anal. Math. Phys. 1 (2011) 139-145.

[49] P. Macklin and J. Lowengrub, An improved geometry-aware curvature discretization for level set methods: application to tumor growth. J. Comput. Phys. 215 (2006) 392-401.

[50] O. Marchal and M. Cafasso, Double-scaling limits of random matrices and minimal $(2 m, 1)$ models: the merging of two cuts in a degenerate case. J. Stat. Mech. Theory Exp. 2011 (2011) P04013.

[51] A. Marshakov, P. Wiegmann and A. Zabrodin, Integrable structure of the Dirichlet boundary problem in two dimensions. Commun. Math. Phys. 227 (2002) 131-153.

[52] A. Martinez-Finkelshtein, P. Martinez-González and R. Orive, Asymptotics of polynomial solutions of a class of generalized Lamé differential equations. Electr. Trans. Numer. Anal. 19 (2005) 18-28.

[53] A. Martínez-Finkelshtein and E.A. Rakhmanov. Critical measures, quadratic differentials, and weak limits of zeros of Stieltjes polynomials. Comm. Math. Phys. 302 (2011) 53-111.

[54] A. Martínez-Finkelshtein and E.B. Saff, Asymptotic properties of Heine-Stieltjes and Van Vleck polynomials. J. Approx. Theory, 118 (2002) 131-151.

[55] E. Memin and P. Perez, Fluid motion recovery by coupling dense and parametric vector fields. IEEE CVPR (1999) 620-625.

[56] H.N. Mhaskar and E.B. Saff, Where does the sup norm of a weighted polynomial live? (A generalization of incomplete polynomials). Constr. Approx. 1 (1985) 71-91.

[57] M.B. Mineev, A finite polynomial solution of the two-dimensional interface dynamics. Physica D 43 (1990) $288-292$.

[58] M. Mineev-Weinstein, Multidimensional pattern formation has an infinite number of constants of motion. Phys. Rev. E $\mathbf{4 7}$ (1993) R2241-R2244.

[59] M.B. Mineev-Weinstein, Selection of the Saffman-Taylor finger in the absence of surface tension: an exact result. Phys. Rev. Lett. 80 (1998) 2113-2116.

[60] M.B. Mineev-Weinstein and S.P. Dawson, A class of non-singular exact solutions for Laplacian pattern formation. Phys. Rev. E 50 (1994) R24-R27.

[61] M. Mineev-Weinstein, P.B. Wiegmann and A. Zabrodin, Integrable structure of interface dynamics. Phys. Rev. Lett. 84 (2000) 5106.

[62] M. Mineev-Weinstein, M. Putinar, L Sander and A. Zabrodin, eds, Physics and mathematics of growing interfaces. Physica $D(2007) 235$.

[63] M. Mineev-Weinstein, M. Putinar and R. Teodorescu, Random matrices in 2D Laplacian growth and operator theory. J. Phys. A: Math. Theor. 41 (2008) 263001. 
[64] M.Y. Mo, The Riemann-Hilbert approach to double scaling limit of random matrix eigenvalues near the "birth of a cut" transition. Int. Math. Res. Not. IMRN 51 (2008) rnn042.

[65] J. Norris and A. Turner, Hastings-Levitov aggregation in the small-particle limit. Commun. Math. Phys. 316 (2012) 809-841.

[66] R. Orive and Z. García, On a class of equilibrium problems in the real axis. J. Comput. Appl. Math. 235 (2010) $1065-1076$.

[67] P. Ya. Polubarinova-Kochina, On a problem of the motion of the contour of a petroleum shell. Dokl. Akad. Nauk USSR 47 (1945) 254-257 (in Russian); English transl., On the displacement of the oil-bearing contour. C. R. (Dokl.) Acad. Sci. URSS 47 (1945) 250-254.

[68] Ch. Pommerenke, Univalent functions, with a chapter on quadratic differentials by G. Jensen. Vandenhoeck \& Ruprecht, Göttingen (1975).

[69] Ch. Pommerenke, Boundary behaviour of conformal maps. Springer, Berlin (1992).

[70] O. Praud and H.L. Swinney, Fractal dimensions and unscreened angles measured for radial viscous fingering. Phys. Rev. E 72 (2005) 011406.

[71] A. Pressley and G. Segal, Loop groups, Oxford Mathematical Monographs. The Clarendon Press, Oxford University Press, New York (1986).

[72] G. Prokert, Existence results for Hele-Shaw flow driven by surface tension. Eur. J. Appl. Math. 9 (1998) 195-221.

[73] E.A. Rakhmanov, The convergence of diagonal Padé approximants. Mat. Sb. (N.S.) 104 (1977) 271-291, 335. English translation: Math. USSR-Sb. 33 (1977) 243-260.

[74] S. Richardson, Hele-Shaw flows with a free boundary produced by the injection of fluid into a narrow channel. J. Fluid Mech. 56 (1972) 609-618.

[75] P.G. Saffman and G. Taylor, The penetration of a fluid into a porous medium or Hele-Shaw cell containing a more viscous liquid. Proc. Roy. Soc. London. Ser. A 245 (1958) 312-329.

[76] E.B. Saff and V. Totik, Logarithmic Potentials with External Fields. Vol. 316 of Grundlehren der Mathematischen Wissenschaften. Springer-Verlag, Berlin (1997).

[77] Y. Sawada, A. Dougherty and J.P. Gollub, Dendritic and fractal patterns in electrolytic metal deposits. Phys. Rev. Lett. 56 (1986) 1260-1263.

[78] B. Shraiman and D. Bensimon, Singularities in nonlocal interface dynamics. Phys. Rev. A 30 (1984) 2840.

[79] M.G. Stepanov and L.S. Levitov, Laplacian growth with separately controlled noise and anisotropy. Phys. Rev. E 63 (2001) 061102 .

[80] K. Takasaki and T. Takebe, Integrable hierarchies and dispersionless limit. Rev. Math. Phys. 7 (1995) 743.

[81] G. Taylor and P.G. Saffman, A note on the motion of bubbles in a Hele-Shaw cell and porous medium. Q. J. Mech. Appl. Math. 12 (1959) 265-279.

[82] R. Teodorescu, Generic critical points of normal matrix ensembles. J. Phys. A: Math. Theor. 39 (2006) 8921.

[83] R. Teodorescu, E. Bettelheim, O. Agam, A. Zabrodin and P. Wiegmann, Normal random matrix ensemble as a growth problem. Nucl. Phys. B 704 (2005) 407-444.

[84] R. Teodorescu, P. Wiegmann and A. Zabrodin, Unstable fingering patterns of Hele-Shaw flows as a dispersionless limit of the Kortweg-de Vries hierarchy. Phys. Rev. Lett. 95 (2005) 044502.

[85] Y. Tu, Saffman-Taylor problem in sector geometry: solution and selection. Phys. Rev. A 44 (1991) 1203-1210.

[86] W. Van Assche, J. Geronimo and A.B.J. Kuijlaars, Riemann-Hilbert problems for multiple orthogonal polynomials, in Special Functions 2000 edited by J. Bustoz et al. Kluwer, Dordrecht (2001) 23-59.

[87] A.N. Varchenko and P.I. Etingof, Why the boundary of a round drop becomes a curve of order four. Vol. 3 of University Lecture Series. American Mathematical Society, Providence, RI (1992).

[88] Yu.P. Vinogradov and P.P. Kufarev, On some particular solutions of the problem of filtration. Doklady Akad. Nauk SSSR (N.S.) 57 (1947) 335-338.

[89] D.V. Voiculescu, Free probability for pairs of faces II: 2-variables bi-free partial R-transform and systems with rank $\leq 1$ commutation. Ann. Inst. Henri Poincaré Probab. Statist. 52 (2016) 1-15.

[90] P.B. Wiegmann and A. Zabrodin, Conformal maps and integrable hierarchies. Commun. Math. Phys. 213 (2000) $523-538$.

[91] P. Wiegmann and A. Zabrodin, Large scale correlations in normal and general non-Hermitian matrix ensembles. J. Phys. A 36 (2003) 3411-3424.

[92] P. Wiegmann and A. Zabrodin, Large N expansion for normal and complex matrix ensembles. Frontiers Number Theory, Physics, and Geometry I. Springer, Berlin/Heidelberg, Part I (2006) 213-229. 